\section{A length-dependent evolutionarily conserved pathway controls nuclear export of circular RNAs}

\author{
Chuan Huang, Dongming Liang, \\ Deirdre C. Tatomer, and Jeremy E. Wilusz
}

Department of Biochemistry and Biophysics, University of Pennsylvania Perelman School of Medicine, Philadelphia, Pennsylvania 19104, USA

Circular RNAs (circRNAs) are generated from many protein-coding genes. Most accumulate in the cytoplasm, but how circRNA localization or nuclear export is controlled remains unclear. Using RNAi screening, we found that depletion of the Drosophila DExH/D-box helicase Hel25E results in nuclear accumulation of long (>800nucleotide), but not short, circRNAs. The human homologs of Hel25E similarly regulate circRNA localization, as depletion of UAP56 (DDX39B) or URH49 (DDX39A) causes long and short circRNAs, respectively, to become enriched in the nucleus. These data suggest that the lengths of mature circRNAs are measured to dictate the mode of nuclear export.

Supplemental material is available for this article.

Received March 26, 2018; revised version accepted April 24, 2018.

As most eukaryotic genes are arranged in a split manner, nascent transcripts must be spliced so that each of the noncoding intronic segments are removed and the exonic segments are joined to one another. This step in gene expression is highly regulated and can be exploited to generate a diverse set of RNAs, including circular RNAs (circRNAs) that have covalently linked ends (for reviews, see Barrett and Salzman 2016; Ebbesen et al. 2016; Wilusz 2018). circRNAs are generated from many protein-coding genes when the pre-mRNA splicing machinery "backsplices" to join a splice donor to an upstream splice acceptor (e.g., joining the end of exon 2 to the beginning of exon 2). Backsplicing is often initiated when complementary sequences from different introns base-pair and bring the intervening splice sites close together (Liang and Wilusz 2014; Zhang et al. 2014), but circRNA levels are also controlled by the combinatorial action of RNA-binding proteins (Ashwal-Fluss et al. 2014; Conn et al. 2015; Kramer et al. 2015), exon-skipping events (Barrett et al. 2015), and the availability of spliceosomal components (Liang et al. 2017). As circRNAs are naturally resistant to degradation by exonucleases, some accumulate to much higher levels than their associated linear mRNAs (Salzman et al. 2012). The functions of most circRNAs

[Keywords: circRNA; Hel25E; UAP56; URH49; DDX39A; DDX39B] Corresponding author: wilusz@pennmedicine.upenn.edu

Article published online ahead of print. Article and publication date are online at http://www.genesdev.org/cgi/doi/10.1101/gad.314856.118. remain unknown, but some can act as microRNA sponges (Hansen et al. 2013; Memczak et al. 2013) or templates for translation (for review, see Tatomer and Wilusz 2017).

Although some circRNAs have been reported to accumulate in the nucleus (Li et al. 2015; Conn et al. 2017), most are efficiently exported to the cytoplasm (Salzman et al. 2012; Jeck et al. 2013). Considering that many circRNAs are expressed in nondividing cells, including neurons, it seems highly likely that active transport processes (and not simply a reliance on nuclear envelope breakdown during mitosis) regulate the localization of circRNAs. However, no factors involved in circRNA nuclear export have been identified so far.

RNAs typically reach the cytoplasm by binding to export receptors that enable transport through nuclear pore complexes (for reviews, see Cullen 2003; Stutz and Izaurralde 2003; Kohler and Hurt 2007; Natalizio and Wente 2013). To export classes of RNAs with highly related structures (e.g., transfer RNAs [tRNAs] or microRNAs), classspecific receptors known as karyopherins or importins/ exportins directly bind these transcripts. In contrast, mRNAs differ significantly in length, sequence, and structure, so bulk mRNA export mechanisms take advantage of adaptor proteins-such as Aly/REF or SR (serine/argininerich) proteins-that are loaded cotranscriptionally onto the mRNA, often in the context of larger protein complexes such as the transcription/export (TREX), THO, and/or exon junction (EJC) complexes. These adaptor proteins then recruit the heterodimeric mRNA export receptor NXF1-NXT1 (also known as TAP-p15 in metazoans) to enable transport through the nuclear pore. Here, we used RNAi screening to identify factors that regulate nuclear export of circRNAs in Drosophila and human cells. We found that Drosophila Hel25E and its human homologs, UAP56/URH49, are key modulators of circRNA localization and control the efficiency of nuclear export by sensing the lengths of mature circRNAs.

\section{Results and Discussion}

Depletion of the DExH/D-box helicase Hel25E results in nuclear accumulation of circdati

To examine the localization of circRNAs in Drosophila DL1 cells, we used biochemical fractionation to isolate nuclear and cytoplasmic RNAs followed by quantitative RT-PCR (qRT-PCR) or Northern blotting. circRNAs derived from the laccase 2 and dati genes were found to localize predominately to the cytoplasm regardless of whether they were generated from their endogenous gene loci (Fig. 1A) or an expression plasmid (Fig. 1B; Supplemental Fig. $\mathrm{S} 1 \mathrm{~A}, \mathrm{~B})$. We reasoned that RNAi screening could be used to reveal factors that control circRNA localization, and thus 26 proteins with known roles in nuclear export of various classes of RNAs were individually knocked down in DL1 cells for $3 \mathrm{~d}$ using dsRNAs (Supplemental

(C) 2018 Huang et al. This article is distributed exclusively by Cold Spring Harbor Laboratory Press for the first six months after the full-issue publication date (see http://genesdev.cshlp.org/site/misc/terms.xhtml). After six months, it is available under a Creative Commons License (Attribution-NonCommercial 4.0 International), as described at http://creativecommons.org/licenses/by-nc/4.0/. 

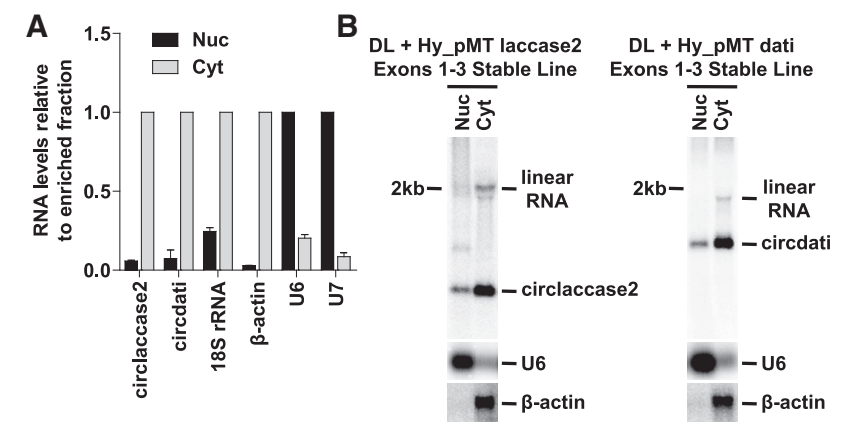

C

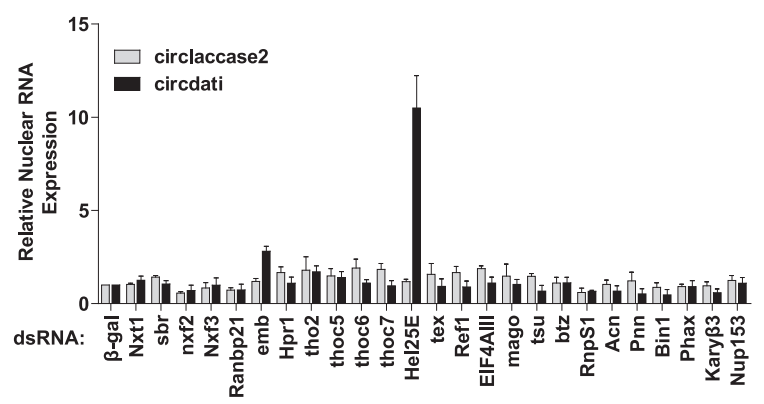

D

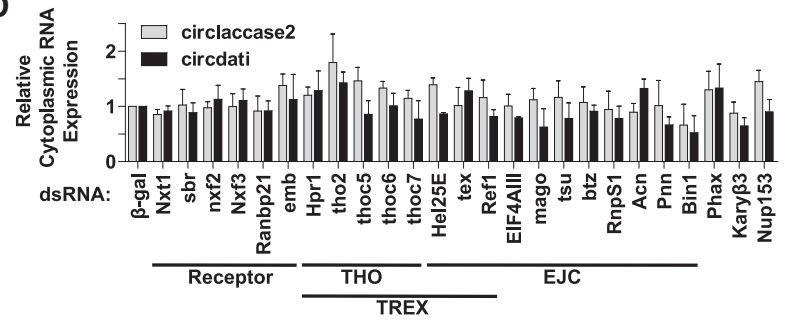

Figure 1. Drosophila circRNAs are enriched in the cytoplasm but can accumulate in the nucleus upon depletion of Hel25E. (A) qRT-PCR quantification of endogenous Drosophila circRNAs in RNA purified from DL1 nuclear (Nuc) or cytoplasmic (Cyt) fractions. U6 and U7 snRNAs served as markers for the nuclear fraction. $18 \mathrm{~S}$ ribosomal RNA (rRNA) and $\beta$-actin mRNA served as markers for the cytoplasmic fraction. The enriched fraction is set to 1 . Data are shown as mean \pm SD. $n=3$. (B) DL1 cell lines stably expressing an inducible laccase2 (left) or dati (right) reporter plasmid were fractionated after $14 \mathrm{~h}$ of copper sulfate treatment to isolate nuclear and cytoplasmic RNA. Northern blots using $3 \mu \mathrm{g}$ of each RNA fraction were then used to examine the localization of reporter-derived transcripts. $(C, D)$ DL1 cells were treated with the indicated dsRNAs for $3 \mathrm{~d}$ followed by purification of nuclear $(C)$ and cytoplasmic $(D)$ RNA fractions. qRT-PCR was used to quantify expression of endogenous circlaccase2 and circdati. Data were normalized to the $\beta$-gal dsRNA sample and are shown as mean \pm SD. $n=3$. Known nuclear export receptors as well as components of the THO, TREX, and EJC are noted.

Fig. S1C). qRT-PCR was then used to measure steadystate circRNA levels in the nuclear and cytoplasmic fractions (Fig. 1C,D). Depletion of nuclear export factor (NXF) family members Nxt1 or sbr (also known as NXF1) had no effect on steady-state circRNA levels or localization but did result in nuclear accumulation of heat-shock factor mRNAs, as reported previously (Supplemental Fig. S1D; Herold et al. 2001; Wilkie et al. 2001). We instead found that depletion of the DExH/D-box helicase Hel25E resulted in nuclear accumulation of the 1120-nucleotide (nt) circdati transcript (Fig. 1C). This phenotype was not due to an overall change in total circdati expression (Supplemental Fig. S2) and was robust, since it was (1) observed using an independent nonoverlapping Hel25E dsRNA
(Supplemental Fig. S3A-C), (2) rescued using an Hel25E expression plasmid that is insensitive to the dsRNA (Supplemental Fig. 3D-F), and (3) also observed when examining plasmid-derived circdati (Supplemental Fig. S4A). Steady-state levels of circdati in the cytoplasm decreased only slightly upon Hel25E depletion (Fig. 1D), likely due to the long half-lives of circRNAs and minimal turnover of circRNAs generated prior to dsRNA treatment. Indeed, when cells were treated with 4-thiouridine $(4 \mathrm{sU})$ so that nascent circRNAs could be purified, we found that newly generated circdati transcripts accumulated in the nucleus and largely failed to reach the cytoplasm in the absence of Hel25E (Fig. 2A,B). As Hel25E is required for nuclear export of many Drosophila mRNAs, including heat-shock factor mRNAs (Supplemental Figs. S1D, S5A; Gatfield et al. 2001; Herold et al. 2003), we concluded that efficient nuclear export of circdati requires a subset of factors required for linear mRNA export.

Only long Drosophila circRNAs (>800 nt) require Hel25E for nuclear export

Upon examining the localization of circlaccase2, we surprisingly found that this 490-nt circRNA did not accumulate in the nucleus when Hel25E was depleted (Fig. 1C; Supplemental Fig. S4B). We thus asked whether Hel25E plays a general or limited role in circRNA export by depleting Hel25E from DL1 cells, labeling/purifying nascent RNAs, and then examining the localization of 12 additional circRNAs that were of varying lengths and exon counts (Supplemental Table S1). Notably, half of the nascent circRNAs accumulated significantly in the nucleus upon Hel25E depletion (and were concomitantly depleted from the cytoplasm), while the localization of the other circRNAs was unchanged (Fig. 2A,B). Similar results were observed upon examining the steady-state pools of these circRNAs, although the effects were dampened due to the pool of pre-existing circRNAs, as expected (Supplemental Fig. S5B-D). There was no correlation between nuclear accumulation and number of exons in the mature circRNA. Instead, all of the circRNAs that accumulated in the nucleus were $>811 \mathrm{nt}$ in length, whereas all of the Hel25E-independent transcripts were $<702$ nt (Fig. 2A,B). This suggested that the length of the mature circRNA may dictate the nuclear export mechanism, with Hel25E being required for efficient export of circRNAs $>800 \mathrm{nt}$.

To directly test this length-dependent export model, we generated expression plasmids that produce mature circRNAs of different lengths $(500,700,900,1100$, and 1677 nt) derived from the firefly luciferase gene (Fig. 2C; Supplemental Fig. S6). In all cases, the introns flanking circdati were used to drive the backsplicing reaction. Regardless of the circfirefly exon length, depletion of Hel25E resulted in an $\sim 20 \%$ decrease in total expression of the mature circRNAs (Supplemental Fig. S6A,B). Nevertheless, as the length of the circfirefly exon progressively increased, a larger and larger amount of the transcript accumulated in the nucleus when Hel25E was depleted (Fig. 2C; Supplemental Fig. S6C,D). The localization of short circfirefly transcripts (500 or $700 \mathrm{nt}$ ) was unaffected, while circfirefly transcripts that were $\geq 900 \mathrm{nt}$ accumulated in the nucleus. These data mirror the effects of Hel25E depletion on the localization of endogenous circRNAs (Fig. 2A). We thus conclude that export of Drosophila circRNAs appears to become more and more reliant on Hel25E as the length 
A
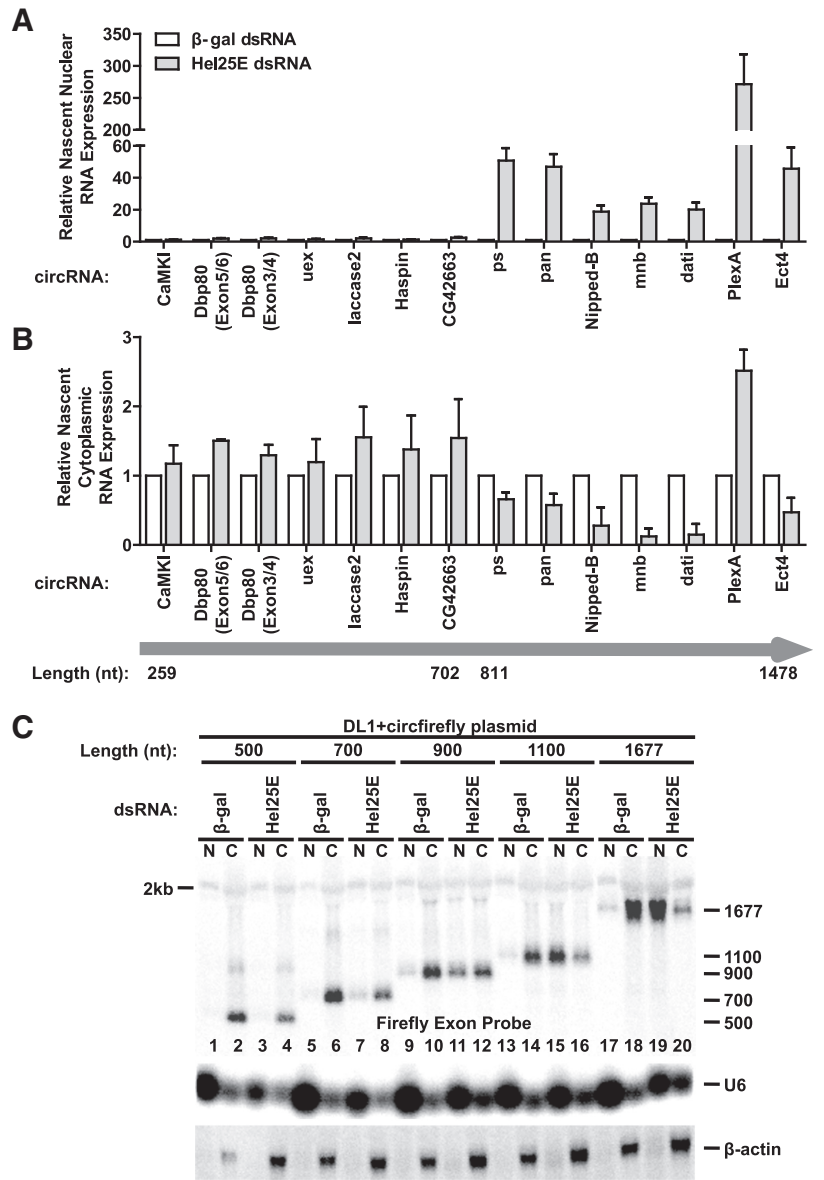

Figure 2. Long (>800-nt) Drosophila circRNAs accumulate in the nucleus upon Hel25E depletion. $(A, B)$ DL1 cells were treated for $3 \mathrm{~d}$ with a control ( $\beta$-gal) dsRNA or a dsRNA to deplete Hel25E and then labeled with $250 \mu \mathrm{M} 4 \mathrm{sU}$ for 15 min prior to isolating RNA from the nuclear $(A)$ and cytoplasmic $(B)$ fractions. 4sU-labeled nascent RNAs were purified from each fraction followed by qRT-PCR to quantify expression of the indicated circRNAs, which are ordered according to their mature circRNA lengths. Data were normalized to the $\beta$-gal dsRNA sample and are shown as mean \pm SD. $n=3$. $(C)$ Reporter plasmids that produce firefly luciferase circRNAs (circfirefly) of different lengths were transfected into DL1 cells treated with the indicated dsRNAs. RNA was then purified from nuclear $(\mathrm{N})$ or cytoplasmic $(C)$ fractions, and $3.6 \mu \mathrm{g}$ of each fraction was run on Northern blots to examine localization of reporter-derived transcripts.

of the transcript increases (Fig. 2C). It is nevertheless important to note that there may be Hel25E-independent modes of circRNA export given that the transcripts did not become fully nuclear-retained when Hel25E was depleted.

\section{UAP56 and URH49, homologs of Drosophila Hel25E, control localization of human circRNAs}

Considering that endogenous human circRNAs also predominately localize to the cytoplasm (Fig. 3A), we next asked whether the human homologs of Hel25E likewise impact circRNA localization patterns. Humans encode two homologs of Hel25E-UAP56 (DDX39B) and URH49 (DDX39A) - that are 90\% identical to one another (Pryor et al. 2004; Yamazaki et al. 2010), with most of the variation occurring within the first 30 amino acids (Fig. 3B; Supplemental Fig. S7). UAP56 and URH49 have been implicated previously in nuclear export of many human mRNAs (Luo et al. 2001; Kapadia et al. 2006; Yamazaki et al. 2010; Akef et al. 2013), and we confirmed that Hsp70 mRNA accumulates in the nucleus after UAP56 or URH49 have been depleted by siRNAs for $48 \mathrm{~h}$ (Fig. 3C; Supplemental Fig. S8A). We then used qRT-PCR to examine the steady-state localization of 14 endogenous human circRNAs that were of varying lengths and exon counts (Fig. 3D,E; Supplemental Table S1; Supplemental Fig. S8B). circRNAs $<356 \mathrm{nt}$ in length accumulated in the nucleus upon depletion of URH49, but their localization was notably unaffected by depletion of UAP56 (Fig. $3 \mathrm{D}, \mathrm{E}$, left panels). In stark contrast, depletion of URH49 did not affect the nuclear levels of circRNAs $>1298 \mathrm{nt}$ in length (Fig. 3D,E, right panels). Instead, these long circRNAs accumulated in the nucleus upon depletion of UAP56. For circRNAs between 411 and 1099 nt in length
A

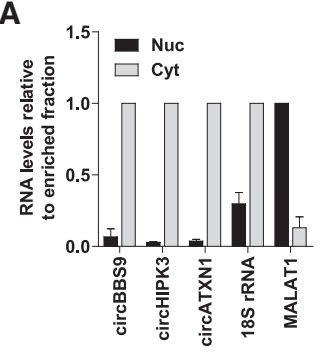

B $83 \%\left[\begin{array}{l}\text { Drosophila Hel25E } \\ \text { Human UAP56 } \\ \text { Human URH49 }\end{array}\right] 85 \%$

C

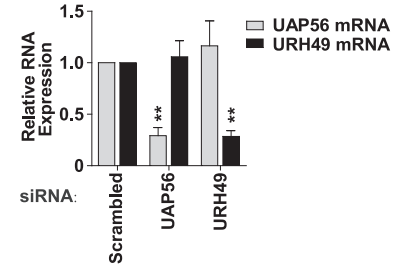

D

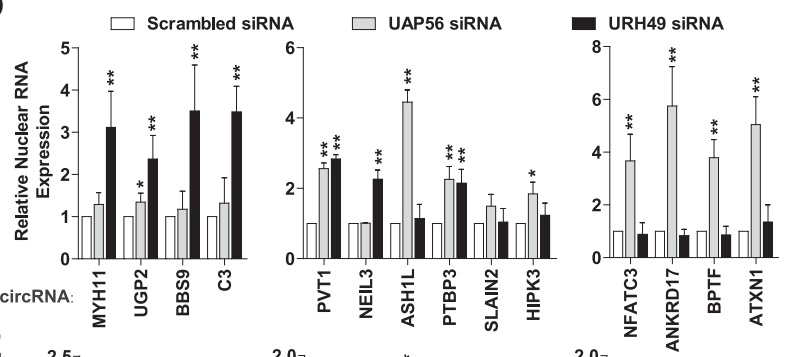

E
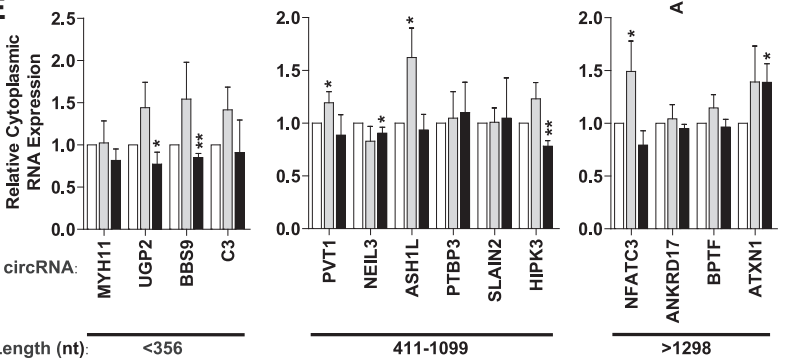

Figure 3. Localization of long and short human circRNAs is controlled by UAP56 and URH49, respectively. (A) qRT-PCR quantification of endogenous human circRNAs in RNA purified from HeLa nuclear (Nuc) or cytoplasmic (Cyt) fractions. MALAT1 and 18S rRNA served as markers for the nuclear and cytoplasmic fractions, respectively. The enriched fraction is set to 1 . (B) Humans encode two closely related homologs of Drosophila Hel25E. The percent amino acid identity is shown. $(C)$ HeLa cells were treated with siRNAs for $48 \mathrm{~h}$ to specifically deplete UAP56 or URH49. $(D, E)$ HeLa cells were then fractionated to isolate nuclear $(D)$ and cytoplasmic $(E)$ RNA. qRT-PCR was used to quantify steady-state expression of the indicated circRNAs, which are ordered according to their mature RNA lengths. Data throughout the figure were normalized to the $\beta$-gal dsRNA samples and are shown as mean \pm SD. $n=3$. $\left({ }^{* *}\right) P<0.01 ;\left({ }^{*}\right) P<0.05$. 
(Fig. 3D,E, middle panels), the regulation appeared to be more complicated, likely because RNA secondary structures impact the overall compactness of the mature circRNA (Masuyama et al. 2004). In total, these data argue that the lengths of mature circRNAs are also measured in human cells, with URH49 and UAP56 modulating the localization of short (<400-nt) and long (>1200-nt) circRNAs, respectively. Consistent with this model, UAP56 and URH49 were found previously by ChIRP-MS (comprehensive identification of RNA-binding proteins by mass spectrometry) to bind to circRNAs in human cells (Chen et al. 2017).

\section{Four amino acids that are divergent among the Hel25E} homologs control their circRNA length preferences

Hel25E and its human homologs have well-characterized ATPase and RNA helicase motifs (Fig. 4A; Shen et al. 2007; Kota et al. 2008; Thomas et al. 2011), but it was unclear how these proteins selectively regulate the localization of circRNAs of different lengths. To define the key

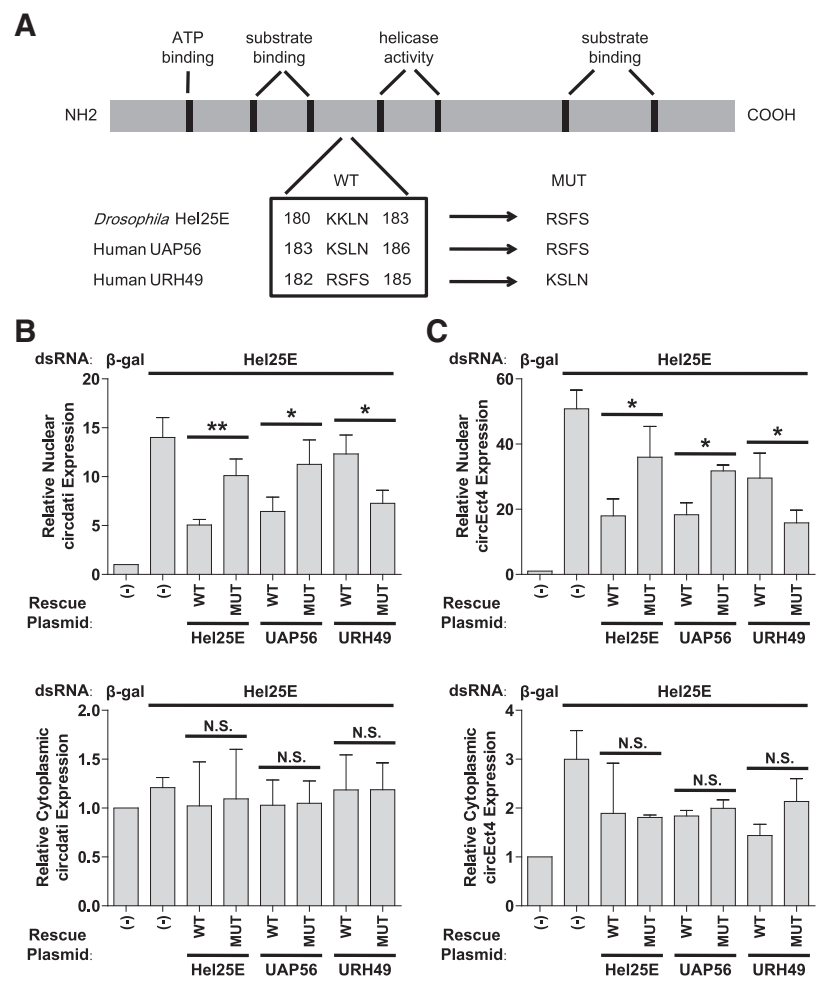

Figure 4. A region divergent among the Hel25E homologs controls their circRNA length preferences. $(A)$ Domain structure of Hel25E family members, highlighting a four-amino-acid region that is divergent between human UAP56 and URH49. Expression plasmids were generated with these amino acids mutated to the indicated sequences. $(B, C) \mathrm{DL} 1$ cells were first treated with a control $(\beta$-gal) dsRNA or a dsRNA to deplete endogenous Hel25E. On the next day, inducible plasmids expressing wild-type (WT) or mutant (MUT) helicases (insensitive to dsRNA treatment) were transfected. Copper sulfate $(500 \mu \mathrm{M})$ was added after $8 \mathrm{~h}$ to induce helicase expression from the plasmids, and cells were fractionated after $24 \mathrm{~h}$. qRT-PCR was then used to quantify steady-state expression of endogenous circdati $(B)$ or circEct4 $(C)$ in nuclear and cytoplasmic fractions. Data were normalized to the $\beta$-gal dsRNA sample and are shown as mean \pm SD. $n=3$. (**) $P<0.01 ;^{*}$ ( $^{*} P<0.05$. regulatory motifs, a dsRNA was used to deplete endogenous Hel25E from DL1 cells, and we then attempted to rescue the circRNA nuclear localization phenotypes by expressing mutant versions of Hel25E from plasmids. In cells expressing Hel25E mutants with greatly reduced ATP-binding affinity (K91N) or helicase activity (D193E), four out of the five long circRNAs examined were efficiently exported to the cytoplasm (Supplemental Fig. S9). This suggests that the enzymatic functions of Hel25E are largely dispensable for long circRNA export, a result that is consistent with prior data on the mechanism of linear mRNA export in Schizosaccharomyces pombe (Thakurta et al. 2007).

We next reasoned that the amino acids that control circRNA length preferences should be conserved between Hel25E and UAP56 (which act on long circRNAs) but not conserved in URH49 (which acts on short circRNAs). We identified a four-amino-acid motif located between the substrate-binding and helicase domains that fits these characteristics: A K-K/S-L-N motif is present in Hel25E/ UAP56, whereas a R-S-F-S motif is present in URH49 (Fig. 4A; Supplemental Fig. S7). To test whether these four amino acids do indeed impact the circRNA length preferences, we generated plasmids expressing mutant Hel25E/UAP56 protein with the URH49 motif and mutant URH49 protein with the Hel25E/UAP56 motif (Fig. 4A). As described above, endogenous Hel25E was depleted from DL1 cells, and the rescue plasmids were then transfected (Fig. 4B,C; Supplemental Fig. S10A). Consistent with expectations, wild-type Hel25E or UAP56 significantly rescued nuclear accumulation of multiple long circRNAs, including circdati (Fig. 4B), circEct4 (Fig. 4C), and circmnb (Supplemental Fig. S10B), but wild-type URH49 did not. Nevertheless, mutating URH49 so that it contained the Hel25E/UAP56 motif was sufficient to allow the protein to rescue the long circRNA nuclear export defects. Likewise, mutating Hel25E or UAP56 so that they contained the URH49 motif caused the proteins to no longer rescue nuclear accumulation of long circRNAs (Fig. 4B,C; Supplemental Fig. S10B). These data clearly demonstrate that the divergent four-amino-acid motif is both necessary and sufficient to dictate the circRNA length preferences of Hel25E homologs.

In summary, we demonstrated that circRNA localization patterns are actively controlled by Hel25E homologs in both Drosophila (Figs. 1, 2) and human (Fig. 3) cells. Drosophila Hel25E and human UAP56 are required for efficient nuclear export of long circRNAs, whereas human URH49 controls the localization of short circRNAs. It remains unclear what factors control localization of short Drosophila circRNAs, but there may be functionally redundant factors and/or other DExH/D-box helicases involved. A role for RNA length in controlling nuclear export of circRNAs was unexpected but is reminiscent of the mechanism that determines how $\mathrm{m}^{7} \mathrm{G}$-capped RNAs are exported (Masuyama et al. 2004; McCloskey et al. 2012). Capped snRNAs are typically short (<200 nt) and exported via the PHAX/CRM1-mediated pathway but become exported via NXF1-NXT1 when their length is increased (regardless of where the extra sequence is inserted). Likewise, long capped mRNAs are typically exported via the NXF1-NXT1 export receptor, but shortening intronless mRNAs causes them to be exported via PHAX/CRM1 (Masuyama et al. 2004). Heterogeneous nuclear ribonucleoprotein (hnRNP) C1/C2 binds to regions in $\mathrm{m}^{7} \mathrm{G}$-capped transcripts that are $>200-300 \mathrm{nt}$ and acts to 
competitively block PHAX binding, thereby committing long capped transcripts to the NXF1-NXT1 pathway (McCloskey et al. 2012). It is tempting to speculate that an analogous mechanism may be used to measure circRNA lengths, but further work is required to define the molecular details. Regardless of the exact mechanism, circRNA lengths are somehow communicated to the Hel25E homologs, perhaps directly via the divergent four-amino-acid motif (Fig. 4), and these proteins then enable efficient nuclear export of the mature circRNAs. Previous work has suggested that UAP56 and URH49 have distinct proteinbinding partners, and it is possible that these unique interactions may play distinct roles in circRNA export. Interestingly, it has also been proposed that UAP56 and URH49 preferentially export distinct subsets of linear mRNAs (Yamazaki et al. 2010), but a role for mRNA length was not examined. Our work suggests that RNA lengths may be measured more often than is currently appreciated, and it is thus possible that a similar mechanism involving these helicases may be used to distinguish mRNAs of different lengths. In conclusion, although circRNAs have variable lengths, sequences, and structures, we provided evidence that these transcripts are fed into specific nuclear export pathways by evolutionarily conserved factors that measure the length of the mature circRNAs.

\section{Materials and methods}

Drosophila cell culture, RNAi, and transfections

Drosophila DL1 cells were cultured at $25^{\circ} \mathrm{C}$ in Schneider's Drosophila medium (Thermo Fisher Scientific, 21720024) supplemented with $10 \%$ fetal bovine serum (HyClone, SH30910.03), 1\% (v/v) penicillin-streptomycin (Thermo Fisher Scientific, 15140122), and 1\% (v/v) L-glutamine (Thermo Fisher Scientific, 35050061). DL1 stable cell lines were generated and maintained by selection with $150 \mu \mathrm{g} / \mathrm{mL}$ hygromycin B. dsRNAs from the Drosophila RNAi Screening Center were generated by in vitro transcription (MEGAscript kit, Thermo Fisher Scientific, AMB13345) of PCR templates containing the T7 promoter sequence on both ends. Primer sequences are shown in Supplemental Table S2. RNAi experiments in sixwell dishes were performed by bathing $3 \times 10^{6}$ cells with $4 \mu \mathrm{g}$ of dsRNA. Cells were incubated for $3 \mathrm{~d}$, and, where indicated, a final concentration of $500 \mu \mathrm{M}$ copper sulfate (Fisher BioReagents, BP346-500) was added for $14 \mathrm{~h}$. Cells were then fractionated (as described below), or total RNA was isolated using Trizol (Thermo Fisher Scientific, 15596018) as per the manufacturer's instructions.

To generate plasmids for transfection into Drosophila DL1 cells, the indicated sequences were inserted into a pMK33/pMtHy-based vector between the metallothionein promoter (pMT) and the SV40 polyadenylation signal as described in detail in the Supplemental Material. For transient transfections of plasmids, $2 \times 10^{6}$ cells were plated in complete medium in six-well dishes, and $2 \mu \mathrm{g}$ of each expression plasmid was transfected using Effectene $(16 \mu \mathrm{L}$ of enhancer and $30 \mu \mathrm{L}$ of Effectene reagent; Qiagen, 301427 ). On the following day, a final concentration of $500 \mu \mathrm{M}$ copper sulfate was added for $14 \mathrm{~h}$ (where indicated) to induce transcription from the pMT. For experiments involving both RNAi and transfection of plasmids, $2 \times 10^{6}$ cells were plated in six-well dishes and bathed with $4 \mu \mathrm{g}$ of dsRNA. On the following day, each rescue plasmid was transfected using Effectene, $500 \mu \mathrm{M}$ copper sulfate was added to induce plasmid expression, and RNA/ protein samples were isolated as indicated.

Metabolic labeling of newly transcribed RNA using $4 \mathrm{sU}$ was performed as described previously (Russo et al. 2017) with modifications that are described in detail in the Supplemental Material.

\section{Mammalian cell culture and transfections}

HeLa cells were cultured at $37^{\circ} \mathrm{C}$ and $5 \% \mathrm{CO}_{2}$ with DMEM (Thermo Fisher Scientific, 11995065) supplemented with 10\% fetal bovine serum and $1 \%$ (v/v) penicillin-streptomycin. siRNAs (100 nM final concentration) were transfected into HeLa cells using Lipofectamine RNAiMAX (Thermo Fisher Scientific, 13778150) according to the manufacturer's instructions. Forty-eight hours after siRNA transfection, cells were fractionated (as described below), or total RNA was isolated using Trizol. siRNA sequences are listed in Supplemental Table S2.

\section{Nuclear and cytoplasmic fractionation}

Cellular fractionation was performed as described previously (Topisirovic et al. 2003) with minor modifications. Briefly, cells were washed twice with serum-free medium (DL1 cells) or PBS (HeLa cells) and resuspended with slow pipetting in $1 \mathrm{~mL}$ of lysis buffer $\mathrm{B}(10 \mathrm{mM}$ Tris- $\mathrm{HCl}$ at $\mathrm{pH} 8$, $140 \mathrm{mM} \mathrm{NaCl}, 1.5 \mathrm{mM} \mathrm{MgCl} 2,0.5 \%$ IGEPAL CA-630, $1 \mathrm{mM}$ dithiothreitol, $80 \mathrm{U} / \mathrm{mL}$ RNase inhibitor [New England Biolabs, M0314L]). Samples were centrifuged at $1000 \mathrm{~g}$ for $3 \mathrm{~min}$ at $4^{\circ} \mathrm{C}$, and the supernatant was saved as the cytoplasmic fraction. Pellets were resuspended in $1 \mathrm{~mL}$ of lysis buffer B, and $100 \mu \mathrm{L}$ of detergent $(3.3 \%[\mathrm{w} / \mathrm{v}]$ sodium deoxycholate, $6.6 \%[\mathrm{v} / \mathrm{v}]$ Tween 40 ) was added. Samples were incubated for $5 \mathrm{~min}$ on ice, and nuclei were then pelleted by centrifugation at $1000 \mathrm{~g}$ for $3 \mathrm{~min}$. The supernatant was removed, and the nuclei were washed with $1 \mathrm{~mL}$ of lysis buffer B followed by centrifugation at $1000 \mathrm{~g}$ for $3 \mathrm{~min}$. The final pellet (nuclear fraction) was resuspended in Trizol. Efficient fractionation of nuclear and cytoplasmic RNAs was verified by measuring levels of U6 snRNA and $\beta$ actin mRNA (DL1 cells) or MALAT1 and 18S ribosomal RNA (rRNA; HeLa cells).

\section{$q R T-P C R$}

Total RNA, cytoplasmic RNA, and nuclear RNA fractions were reversetranscribed using random hexamers and SuperScript III (Thermo Fisher Scientific, 18080051) according to the manufacturer's instructions. qRTPCR was then carried out in triplicate using Power SYBR Green PCR master mix (Thermo Fisher Scientific, 4367659). All qRT-PCR primer sequences are in Supplemental Tables S3 and S4.

\section{Northern blotting}

Northern blots using NorthernMax reagents (ThermoFisher Scientific) and oligonucleotide probes were performed as described previously (Tatomer et al. 2017). All probe sequences are in Supplemental Table S5. Blots were viewed with a Typhoon 9500 scanner (GE Healthcare) and quantified using ImageJ. Representative blots are shown.

\section{Western blotting}

Whole-cell protein extracts were prepared using RIPA buffer, separated on NuPAGE 4\%-12\% Bis-Tris gels (Thermo Fisher Scientific, NP0323), and transferred to a PVDF membrane (Bio-Rad, 1620177). Antibodies used were $\alpha$-Flag M2 antibody (1:1,000 dilution; Sigma, F1804), $\alpha$-Tubulin antibody (1:10,000 dilution; Sigma, T6074), and $\alpha$-Discs Large (1:1,000 dilution; Developmental Studies Hybridoma Bank, 4F3). Membranes were processed using a standard ECL protocol (Thermo Fisher Scientific, EI9051).

\section{Statistical analyses}

Statistical significance for comparisons of means was assessed by Student's $t$-test for qRT-PCRs and Northern blots. Statistical details and error bars are defined in each figure legend: $P<0.01\left({ }^{* *}\right)$ and $P<0.05\left({ }^{*}\right)$.

\section{Acknowledgments}

We thank all members of the Wilusz laboratory for discussions and advice. This work was supported by start-up funds from the University of Pennsylvania and by National Institutes of Health grants R00-GM104166 and R35GM119735. J.E.W. is a Rita Allen Foundation Scholar.

Author contributions: C.H. and J.E.W. conceived and designed the project. C.H., D.L., and D.C.T. performed experiments and analyzed data. C.H. and J.E.W. wrote the manuscript with input from the other authors. 


\section{References}

Akef A, Zhang H, Masuda S, Palazzo AF. 2013. Trafficking of mRNAs containing ALREX-promoting elements through nuclear speckles. Nucleus 4: 326-340.

Ashwal-Fluss R, Meyer M, Pamudurti NR, Ivanov A, Bartok O, Hanan M, Evantal N, Memczak S, Rajewsky N, Kadener S. 2014. circRNA biogenesis competes with pre-mRNA splicing. Mol Cell 56: 55-66.

Barrett SP, Salzman J. 2016. Circular RNAs: analysis, expression and potential functions. Development 143: 1838-1847.

Barrett SP, Wang PL, Salzman J. 2015. Circular RNA biogenesis can proceed through an exon-containing lariat precursor. Elife 4: e07540.

Chen YG, Kim MV, Chen X, Batista PJ, Aoyama S, Wilusz JE, Iwasaki A, Chang HY. 2017. Sensing self and foreign circular RNAs by intron identity. Mol Cell 67: 228-238.e5.

Conn SJ, Pillman KA, Toubia J, Conn VM, Salmanidis M, Phillips CA, Roslan S, Schreiber AW, Gregory PA, Goodall GJ. 2015. The RNA binding protein quaking regulates formation of circRNAs. Cell 160: 1125-1134.

Conn VM, Hugouvieux V, Nayak A, Conos SA, Capovilla G, Cildir G, Jourdain A, Tergaonkar V, Schmid M, Zubieta C, et al. 2017. A circRNA from SEPALLATA3 regulates splicing of its cognate mRNA through R-loop formation. Nat Plants 3: 17053.

Cullen BR. 2003. Nuclear RNA export. J Cell Sci 116: 587-597.

Ebbesen KK, Kjems J, Hansen TB. 2016. Circular RNAs: identification, biogenesis and function. Biochim Biophys Acta 1859: 163-168.

Gatfield D, Le Hir H, Schmitt C, Braun IC, Kocher T, Wilm M, Izaurralde E. 2001. The DExH/D box protein HEL/UAP56 is essential for mRNA nuclear export in Drosophila. Curr Biol 11: 1716-1721.

Hansen TB, Jensen TI, Clausen BH, Bramsen JB, Finsen B, Damgaard CK, Kjems J. 2013. Natural RNA circles function as efficient microRNA sponges. Nature 495: 384-388.

Herold A, Klymenko T, Izaurralde E. 2001. NXF1/p15 heterodimers are essential for mRNA nuclear export in Drosophila. RNA 7: 1768-1780.

Herold A, Teixeira L, Izaurralde E. 2003. Genome-wide analysis of nuclear mRNA export pathways in Drosophila. EMBO I 22: 2472-2483.

Jeck WR, Sorrentino JA, Wang K, Slevin MK, Burd CE, Liu J, Marzluff WF, Sharpless NE. 2013. Circular RNAs are abundant, conserved, and associated with ALU repeats. RNA 19: 141-157.

Kapadia F, Pryor A, Chang TH, Johnson LF. 2006. Nuclear localization of poly $(\mathrm{A})^{+}$mRNA following siRNA reduction of expression of the mammalian RNA helicases UAP56 and URH49. Gene 384: 37-44.

Kohler A, Hurt E. 2007. Exporting RNA from the nucleus to the cytoplasm. Nat Rev Mol Cell Biol 8: 761-773.

Kota KP, Wagner SR, Huerta E, Underwood JM, Nickerson JA. 2008. Binding of ATP to UAP56 is necessary for mRNA export. J Cell Sci 121: 1526-1537.

Kramer MC, Liang D, Tatomer DC, Gold B, March ZM, Cherry S, Wilusz JE. 2015. Combinatorial control of Drosophila circular RNA expression by intronic repeats, hnRNPs, and SR proteins. Genes Dev 29: 2168-2182.

Li Z, Huang C, Bao C, Chen L, Lin M, Wang X, Zhong G, Yu B, Hu W, Dai L, et al. 2015. Exon-intron circular RNAs regulate transcription in the nucleus. Nat Struct Mol Biol 22: 256-264.

Liang D, Wilusz JE. 2014. Short intronic repeat sequences facilitate circular RNA production. Genes Dev 28: 2233-2247.

Liang D, Tatomer DC, Luo Z, Wu H, Yang L, Chen LL, Cherry S, Wilusz JE. 2017. The output of protein-coding genes shifts to circular RNAs when the pre-mRNA processing machinery is limiting. Mol Cell 68: 940-954 e943.
Luo ML, Zhou Z, Magni K, Christoforides C, Rappsilber J, Mann M, Reed R. 2001. Pre-mRNA splicing and mRNA export linked by direct interactions between UAP56 and Aly. Nature 413: 644-647.

Masuyama K, Taniguchi I, Kataoka N, Ohno M. 2004. RNA length defines RNA export pathway. Genes Dev 18: 2074-2085.

McCloskey A, Taniguchi I, Shinmyozu K, Ohno M. 2012. hnRNP C tetramer measures RNA length to classify RNA polymerase II transcripts for export. Science 335: 1643-1646.

Memczak S, Jens M, Elefsinioti A, Torti F, Krueger J, Rybak A, Maier L, Mackowiak SD, Gregersen LH, Munschauer M, et al. 2013. Circular RNAs are a large class of animal RNAs with regulatory potency. $\mathrm{Na}$ ture 495: 333-338.

Natalizio BJ, Wente SR. 2013. Postage for the messenger: designating routes for nuclear mRNA export. Trends Cell Biol 23: 365-373.

Pryor A, Tung L, Yang Z, Kapadia F, Chang TH, Johnson LF. 2004. Growthregulated expression and G0-specific turnover of the mRNA that encodes URH49, a mammalian DExH/D box protein that is highly related to the mRNA export protein UAP56. Nucleic Acids Res 32: 1857-1865.

Russo J, Heck AM, Wilusz J, Wilusz CJ. 2017. Metabolic labeling and recovery of nascent RNA to accurately quantify mRNA stability. Methods 120: $39-48$.

Salzman J, Gawad C, Wang PL, Lacayo N, Brown PO. 2012. Circular RNAs are the predominant transcript isoform from hundreds of human genes in diverse cell types. PLoS One 7: e30733.

Shen J, Zhang L, Zhao R. 2007. Biochemical characterization of the ATPase and helicase activity of UAP56, an essential pre-mRNA splicing and mRNA export factor. J Biol Chem 282: 22544-22550.

Stutz F, Izaurralde E. 2003. The interplay of nuclear mRNP assembly, mRNA surveillance and export. Trends Cell Biol 13: 319-327.

Tatomer DC, Wilusz JE. 2017. An unchartered journey for ribosomes: circumnavigating circular RNAs to produce proteins. Mol Cell 66: 1-2.

Tatomer DC, Liang D, Wilusz JE. 2017. Inducible expression of eukaryotic circular RNAs from plasmids. Methods Mol Biol 1648: 143-154.

Thakurta AG, Selvanathan SP, Patterson AD, Gopal G, Dhar R. 2007. The nuclear export signal of splicing factor Uap56p interacts with nuclear pore-associated protein Raelp for mRNA export in Schizosaccharomyces pombe. J Biol Chem 282: 17507-17516.

Thomas M, Lischka P, Muller R, Stamminger T. 2011. The cellular DExD/ H-box RNA-helicases UAP56 and URH49 exhibit a CRM1-independent nucleocytoplasmic shuttling activity. PLoS One 6: e22671.

Topisirovic I, Guzman ML, McConnell MJ, Licht JD, Culjkovic B, Neering SJ, Jordan CT, Borden KL. 2003. Aberrant eukaryotic translation initiation factor 4E-dependent mRNA transport impedes hematopoietic differentiation and contributes to leukemogenesis. Mol Cell Biol 23: 8992-9002.

Wilkie GS, Zimyanin V, Kirby R, Korey C, Francis-Lang H, Van Vactor D, Davis I. 2001. Small bristles, the Drosophila ortholog of NXF-1, is essential for mRNA export throughout development. RNA 7: 17811792.

Wilusz JE. 2018. A 360 degrees view of circular RNAs: from biogenesis to functions. Wiley Interdiscip Rev RNA: e1478.

Yamazaki T, Fujiwara N, Yukinaga H, Ebisuya M, Shiki T, Kurihara T, Kioka N, Kambe T, Nagao M, Nishida E, et al. 2010. The closely related RNA helicases, UAP56 and URH49, preferentially form distinct mRNA export machineries and coordinately regulate mitotic progression. Mol Biol Cell 21: 2953-2965.

Zhang XO, Wang HB, Zhang Y, Lu X, Chen LL, Yang L. 2014. Complementary sequence-mediated exon circularization. Cell 159: 134-147. 


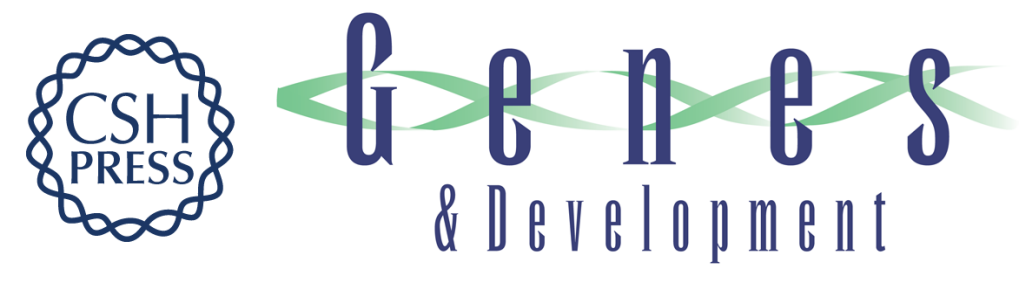

\title{
A length-dependent evolutionarily conserved pathway controls nuclear export of circular RNAs
}

\author{
Chuan Huang, Dongming Liang, Deirdre C. Tatomer, et al. \\ Genes Dev. 2018, 32: originally published online May 17, 2018 \\ Access the most recent version at doi:10.1101/gad.314856.118
}

\section{Supplemental http://genesdev.cshlp.org/content/suppl/2018/05/17/gad.314856.118.DC1 \\ Material}

Related Content

Size matters: conserved proteins function in length-dependent nuclear export of circular RNAs

Yao Wan and Anita K. Hopper

Genes Dev. May , 2018 32: 600-601

References This article cites 39 articles, 15 of which can be accessed free at:

http://genesdev.cshlp.org/content/32/9-10/639.full.html\#ref-list-1

Articles cited in:

http://genesdev.cshlp.org/content/32/9-10/639.full.html\#related-urls

Creative This article is distributed exclusively by Cold Spring Harbor Laboratory Press for the first Commons six months after the full-issue publication date (see

License http://genesdev.cshlp.org/site/misc/terms.xhtml). After six months, it is available under a Creative Commons License (Attribution-NonCommercial 4.0 International), as described at http://creativecommons.org/licenses/by-nc/4.0/.

Email Alerting Receive free email alerts when new articles cite this article - sign up in the box at the top Service right corner of the article or click here.

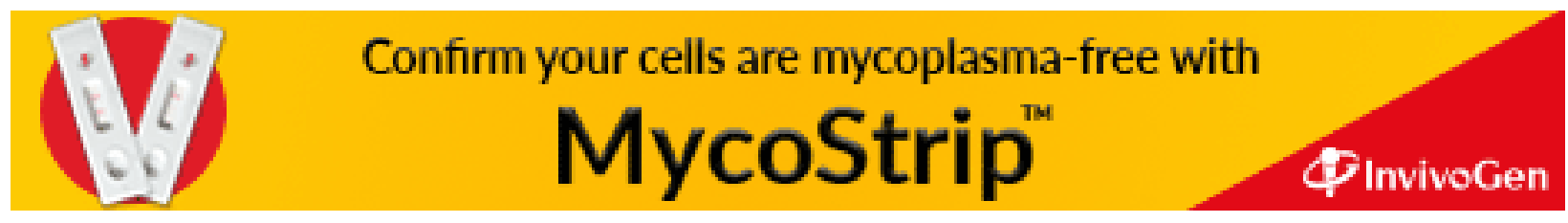

